\title{
Atmospheric Neutrino Fluxes
}

\author{
Thomas K. Gaisser吨

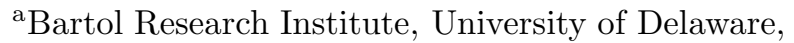 \\ Newark, DE 19716, USA
}

This talk is a status report on calculations of the flux of atmospheric neutrinos from the sub-GeV range to $E_{\nu} \sim \mathrm{PeV}$. In the lower energy range $\left(E_{\nu}<1 \mathrm{TeV}\right)$ the primary interest is in using the atmospheric neutrino beam to study neutrino oscillations. In the $\mathrm{TeV}$ range and above, atmospheric neutrinos are a calibration source and background for neutrino telescopes.

\section{INTRODUCTION}

The discovery of neutrino oscillations with atmospheric neutrinos makes it important to know the production spectrum of neutrinos as precisely as possible in order to infer the properties and parameters of the oscillations from the data. It also means that the flux of cosmic-ray induced neutrinos is much better measured than it otherwise might have been. In addition to the extensive measurements at Super-K, 1 there were important measurements at Soudan [2] and MACRO. [3] The measurements cover a range of energies and techniques, and they see the beam from different locations in the geomagnetic field, which exposes interesting effects at low energy.

Because of oscillations, measuring the cosmicray neutrino beam is an iterative process in which the oscillations and fluxes must be understood from the same data. Fortunately, calculation of the neutrino spectrum at production is straightforward, and it can be checked by comparison to measurements of atmospheric muons. Moreover, the evidence for oscillations is robust because it is based on ratios, which are better known that the absolute normalization of the atmospheric neutrino beam. The anomalous ratio of electronlike to muon like events reveals a relative deficit of $\nu_{\mu}$ at low energy, and the ratio of upward to downward multi-GeV events reflects the pathlength dependence of $\nu_{\mu}$ oscillations and defines a range of $\delta m^{2}$. [1] All this is reinforced by the

\footnotetext{
* Research supported in part by the U.S. Department of Energy under Grant No. DE-FG02-91ER40626
}

low ratio of stopping to throughgoing upward, neutrino-induced muons [4, [5] and by the low ratio of vertically upward to horizontal throughgoing muons. [6,7] A consistent pattern of energy and pathlength dependence emerges that clearly points to neutrino oscillations as the explanation. Because atmospheric $\nu_{e}$ behave normally to the precision measured so far, the main effect must lie in the $\nu_{\mu} \leftrightarrow \nu_{\tau}$ sector (or involve sterile neutrinos, which are now disfavored). 8, 8, 3]

Here I review the main features of the calculation of the atmospheric neutrino beam at production, emphasizing the simple features that provide the basis for the evidence for oscillations. This talk is based to a large extent on a recent review [9]. I organize the material here in order of increasing energy.

The atmospheric neutrino flux is a convolution of the primary spectrum at the top of the atmosphere with the yield $(Y)$ of neutrinos per primary particle. To reach the atmosphere and interact, the primary cosmic rays first have to pass through the geomagnetic field. Thus the flux of neutrinos of type $i$ can be represented as

$$
\begin{aligned}
\phi_{\nu_{i}}= & \phi_{p} \otimes R_{p} \otimes Y_{p \rightarrow \nu_{i}} \\
& +\sum_{A}\left\{\phi_{A} \otimes R_{A} \otimes Y_{A \rightarrow \nu_{i}}\right\},
\end{aligned}
$$

where $\phi_{p(A)}$ is the flux of primary protons (nuclei of mass A) outside the influence of the geomagnetic field and $R_{p(A)}$ represents the filtering effect of the geomagnetic field. Free and bound nucle- 


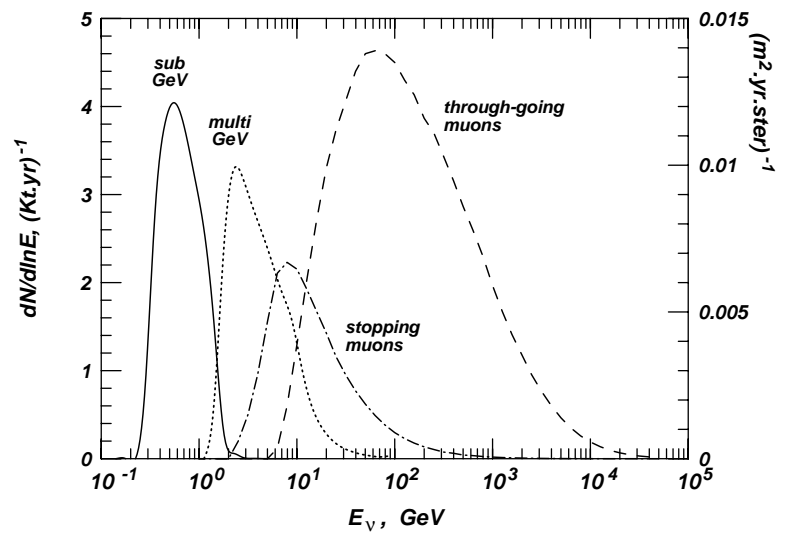

Figure 1. Distribution of neutrino energies that give rise to several classes of neutrino events.

ons are treated separately.

Each of the factors on the right side of Eq. 1 is a potential source of uncertainty. Since the uncertainties depend on energy, one needs an estimate of the relative importance of different primary energies for a given region of neutrino energy. Fig. 1 [10] shows the distributions of neutrino energies for four classes of events. Very roughly, sub-GeV events, multi-GeV events, upward stopping muons and upward throughgoing muons correspond respectively to primary cosmic-ray energies of $10^{1 \pm 0.5}, 10^{1.5 \pm 0.5}, 10^{2.0 \pm 0.5}$ and $10^{3.0 \pm 1} \mathrm{GeV}$. Fig. 2 shows the response function in detail for the sub-GeV events, as defined at Super-K [1].

\section{LOW ENERGIES}

The sub-GeV response is at low enough energy so that it depends strongly on geomagnetic location and to some extent on the state of solar modulation, as illustrated in Fig. 2. The three pairs of curves show the distributions of primary energy per nucleon that produce sub-GeV events under different conditions. These response curves are normalized so that the area under each curve is proportional to the corresponding signal in the absence of oscillations. "S-K downgoing" is for events at Super-K induced by cosmic-rays from above; "up" is for events at Super-K induced by cosmic-rays from below the horizon; and "Nocutoff" is the response in the absence of any geomagnetic field effects. The latter situation is realized in practice for downward events at Soudan and SNO, which have low local geomagnetic cutoffs. The local geomagnetic cutoff at Super-K is high (11.5 GV for vertically downward events), so the rate of downward sub-GeV events at Super$\mathrm{K}$ in the absence of oscillations would be significantly lower than the rate of upward events. This effect is partially washed out by the angle between the direction of the charged lepton, which is measured, and the neutrino that produced it, and it is reversed for muon-like events by the effect of neutrino oscillations [1].

Solar modulation may also have a noticeable effect on rates in a sufficiently large statistical sample. The dashed lines show the maximum effect of solar modulation (corresponding to conditions during the last two years of data taking at Super$\mathrm{K})$. Neutron monitor records give an appropriate measure of solar modulaton because the neutron monitor response is similar to the response of atmospheric neutrinos. The shape of the response function of a high-latitude neutron monitor is indicated in Fig. 2. 11]

\subsection{East-West effect}

This effect arises from the systematic bending of the positively charged primary cosmic rays in the geomagnetic field before they reach the atmosphere to interact. At low geomagnetic latitudes the result is a significantly larger geomagnetic cutoff for particles arriving from the East than for those arriving from the West. Thus the integrated cosmic-ray intensity is greater from the West than from the East, and this asymmetry is reflected by secondary neutrinos and muons. Observation of the East-West effect in muons confirmed long ago that the primary cosmic rays are positive. 12,13 Now observation of this effect with neutrinos at Super-Kamiokande 14] provides an important systematic confirmation of the analysis and interpretation of the evidence for neutrino oscillations. 
Single-ring events with momentum in the interval 0.4 to $3 \mathrm{GeV} / \mathrm{c}$ and zenith angles $(\theta)$ within $30^{\circ}$ of the horizon were compared [14] to calculations [15,16] of the shape of the distribution in azimuth $(\phi)$. The comparison is independent of oscillation effects to first order because the distribution of pathlengths is constant over the $360^{\circ}$ band of $\phi$ with $60^{\circ}<\theta<120^{\circ}$. Thus the agreement between observed and expected shapes shows that geomagnetic effects are under control in the atmospheric neutrino flux calculations, reinforcing confidence in the interpretation of the up-down asymmetry of the flux of muon neutrinos as a consequence of oscillations.

\subsection{Primary spectrum}

An estimate [17] of the uncertainty in the primary spectrum in the light of recent measurements by BESS [18] and AMS [19] is $\pm 5 \%$ below $100 \mathrm{GeV} /$ nucleon, which covers the energies relevant for sub-GeV events. This estimate is based on a fit to BESS and AMS data alone. A more proper estimate of the uncertainty in the primary spectrum would use all valid measurements, including those with larger quoted uncertainties, to estimate the systematic uncertainty in the primary spectrum. Excluding an early measurement 20] which appears to be anomalously high, the measurements cover a range of $\sim+10 \%$ to $-20 \%$ below $100 \mathrm{GeV}$ relative to the measurements of BESS and AMS.

Figs. 3 and 4 illustrate the uncertainty in the neutrino flux from the primary spectrum uncertainty. The plots show the calculations using three fits to the primary spectrum with the same interaction model and cascade calculation [21]. Fig. 5 shows the three assumed spectra. The fit of Agrawal et al. 221] was made before the measurements of BESS, AMS and other recent measurements [22,23] and reflects mainly the earlier measurement [24], which has a lower normalization. The two new fits [17] are dominated by the data from BESS and AMS. The plot shows the all-nucleon spectrum, which includes the nucleons bound in nuclei.

\subsection{Treatment of hadronic interactions}

Atmospheric neutrinos come from decay of pions and kaons produced by interactions of cosmicray hadrons in the atmosphere. The neutrino flux depends primarily on the inclusive cross section for $p+$ air $\rightarrow \pi^{ \pm}+X$. Although pion production is peaked near $x_{F}=0$, because of the steep primary spectrum the most important region of phase space for the convolution 1 is in the forward fragmentation region. For pions the range $0.1<x_{F}<0.5$ has most weight, while for kaons the range extends to somewhat larger $x_{F}$ because of $p \rightarrow \Lambda+K^{+}$.

Event generators used in calculations of atmospheric neutrinos are based on various accelerator data. Especially important are several spectrometer experiments with protons in the momentum range $15-30 \mathrm{GeV} / \mathrm{c}$ incident on light nuclei. Each experiment has statistical and systematic uncertainties. In addition, there are systematic uncertainties associated with extrapolation into unmeasured regions of phase space [10]. Comparison of calculations made with different interaction models using the same primary spectrum gives an indication of the magnitude of uncertainties that arise from treatment of hadronic interactions. Such a comparison is shown in Figs. 6 and 7 made with the primary spectrum of Ref. 21 (heavy solid line in Fig. 5).

Figs. 6 and 7 from Ref. [9] display three independent calculations using five different representations of hadronic interactions. The event generator Target- 1 was used in the Bartol neutrino flux calculation 21]. Target-2.1 is a modified version, which is under development for use in threedimensional calculations. The event generator has been adjusted to give better agreement with the pion data around $X_{l a b}=0.2$. Distributions of leading nucleons have been adjusted to give a better representation of neutron/proton ratios. A preliminary description of this work, which is still in progress, is given in Ref. [25]. HKKM indicates the interaction model used in the earlier calculation of Honda et al. [16]; currently [26] they use the event generator Dpmjet3 [27]. FLUKA refers to the three-dimensional calculation of Battistoni et al. 28]. The differences are primarily due to the different representations of pion production, 
rather than to technical differences.

Differences among the calculations are significantly larger at Soudan than at Kamioka, which points to large differences in the treatment of low energy interactions $(<10 \mathrm{GeV})$, which are mostly below the geomagnetic cutoff at Kamioka. Forthcoming results from the HARP experiment [29] should be helpful in reducing this ambiguity. This should allow a useful, quantitative comparison between Soudan and Kamioka, taking account of the rather different geomagnetic environments.

\subsection{Three-dimensional calculations}

An approximation common to the calculations used to interpret the measurements of atmospheric neutrinos so far is that the neutrinos follow the direction of the primaries that produce them. This approximation becomes questionable at energies low enough so the typical transverse momenta of charged pions are comparable to their longitudinal momenta, i.e. for $E_{\nu}<1 \mathrm{GeV}$. Another aspect of one-dimensional calculations is that the bending of secondary charged particles in the geomagnetic field is also neglected. For a fully three-dimensional calculation, one needs to generate events from an isotropic distribution of primaries over the full primary energy spectrum (from pion production threshold) at a dense grid of locations uniformly distributed over the surface of the Earth, while accounting for the angular dependence of the geomagnetic cutoffs at each location. In that case only a tiny fraction ( $\sim A / R_{\oplus}^{2}$ ) of the neutrinos generated will cross a detector of area $A$. Because the radius of the Earth $R_{\oplus} \gg \sqrt{A}$, a brute force calculation is a challenge.

So far, two types of approximation have been used for three-dimensional calculations. Battistoni et al. 28 neglect bending of secondary particles in the geomagnetic field. In this case, a set of cascades generated at a single location (with no cutoff) can be used to obtain the neutrino flux from all directions. This is done by placing the detector where each neutrino crosses the depth of the detector (once as it enters and once as it exits the Earth). The neutrino is then kept if the direction of the primary is such that it would have passed the geomagnetic field coming from the direction required to make the neutrino pass through the detector at its true location. The other approximation (e.g. 30,31,32) is to make $A$ large. There are also preliminary versions of more ambitious calculations 33,34.

An excess of low energy neutrinos from near the horizon is a characteristic feature of threedimensional calculations. 28 The origin of this geometrical effect is discussed by Lipari. 30] Because it occurs only for low energy neutrinos, however, the excess is washed out by the broad angular distribution of the the charged lepton relative to the neutrino direction.

Associated with the horizontal excess is a small change in the pathlength distribution of low energy neutrinos. Quantitatively, the average pathlength of neutrinos arriving from just above the horizon $\left(80^{\circ}<\theta<90^{\circ}\right)$ is about $10 \%$ lower in the $3 \mathrm{D}$ calculation than in the $1 \mathrm{D}$ approximation for $E_{\nu}=0.3 \mathrm{GeV}$. 31 The suppression for $E_{\nu}=1 \mathrm{GeV}$ is $<5 \%$. This effect, which has not yet been included in analysis of Super-K, could be expected to increase the inferred $\delta m^{2}$ slightly (of order $\sim 1 \%$ to keep the factor $\delta m^{2} \times L / E_{\nu}$ constant while decreasing $L$ by $\sim 10 \%$ for $\sim 10 \%$ of sub-GeV events).

During its test flight on the Space Shuttle, AMS mapped the energy spectrum of low-energy primary cosmic rays around the globe. The data show a substantial flux of protons with energies below the local geomagnetic cutoff. 35. These are secondary protons produced by interactions of cosmic-rays above the cutoff that enter the atmosphere at large zenith angles. Depending on their orientation relative to the geomagnetic field, a large fraction of the sub-cutoff secondaries curve back into space. [36 Such albedo particles may remain trapped for several cycles before they re-enter the atmosphere. As a consequence, the rate per area-solid angle at which they re-enter is correspondingly lower than their flux at $380 \mathrm{~km}$. These features have been evaluated quantitatively by Zuccon et al. [37]. Since both the flux of reentering subcutoff particles and their pion multiplicity is lower than for primaries above the cutoff, their contribution to the neutrino flux should 
be small. It is interesting to note that all subcutoff particles are included in the 1D calculations because all secondaries are assumed to follow the direction of the incident primary. In this respect, the 1D calculation overestimates slightly the contribution of sub-cutoff particles since a few may interact on the way up rather than upon re-entry.

Another consequence of bending of secondary charged particles in the geomagnetic field, in this case involving muons, is a second-order systematic effect on the calculation of the East-West effect. The asymmetry is increased for $\nu_{e}$ and reduced slightly for $\nu_{\mu}$. 38]

\section{HIGHER ENERGY}

For $E_{\nu}>1 \mathrm{GeV}$ the energies of the parent cosmic rays are sufficiently high that geomagnetic effects and solar modulation become unimportant. In addition, the direction of the charged lepton is better aligned with the direction of the neutrino. Thus for multi-GeV events and neutrino-induced muons, in the absence of oscillations, one sees the characteristic enhancement of the neutrino flux from near the horizontal. This is related to the "secant theta effect" familiar from atmospheric muons. It is a consequence of the enhanced decay probability for pions at large zenith angles. The corresponding effect for neutrinos is nicely illustrated in the plots of angular distributions of neutrino events from Super-K. [1]

\subsection{Angular dependence}

An analytic approximation for the differential flux of $\nu_{\mu}+\bar{\nu}_{\mu}$ from decay of pions and kaons displays some important features the neutrino flux.

$$
\begin{aligned}
\frac{d N_{\nu}}{d E_{\nu}} & =\frac{\phi_{N}\left(E_{\nu}\right)}{\left(1-Z_{N N}\right)(\gamma+1)}\left\{\left[\frac{Z_{N \pi}\left(1-r_{\pi}\right)^{\gamma}}{1+B_{\pi \nu} \cos \theta E_{\nu} / \epsilon_{\pi}}\right]\right. \\
& \left.+0.635\left[\frac{Z_{N K}\left(1-r_{K}\right)^{\gamma}}{1+B_{K \nu} \cos \theta E_{\nu} / \epsilon_{K}}\right]\right\}
\end{aligned}
$$

where

$\phi\left(E_{0}\right)=\frac{d N}{d E_{0}}=A \times E_{0}^{-(\gamma+1)}$

is the differential primary spectrum of nucleons of energy $E_{0}$. The neutrino flux in Eq. 2 is proportional to the primary spectrum evaluated at the energy of the neutrino. The constants $r_{i}=m_{\mu}^{2} / m_{i}^{2}$ for $i=(\pi, K)$, and the constants $B_{i}$ depend on hadron attenuation lengths as well as decay kinematics [39].

The critical energy for pions is $\epsilon_{\pi} \approx 115 \mathrm{GeV}$, while for kaons $\epsilon_{K} \approx 850 \mathrm{GeV}$. For $E_{\nu} \ll$ $\epsilon_{\pi} / \cos \theta$ the neutrino spectral index is the same as that of the primary spectrum. For $E_{\nu}>$ $\epsilon_{\pi} / \cos \theta$ the spectrum steepens, first for neutrinos from pion decay and at higher energy $\left(E_{\nu}>\right.$ $\epsilon_{K} / \cos \theta$ ) for neutrinos from decay of kaons. As energy increases so does the horizontal enhancement from the zenith angle dependence in the denominator of Eq. 2. Fig. 8 shows the fraction of atmospheric neutrinos and atmospheric muons that come from pions and from kaons at two angles. Above $\sim 100 \mathrm{GeV}$, kaons dominate as the source of neutrinos. It is the larger value of $\epsilon_{K}$, together with the form of the kinematical factor in the numerator, that determines the behavior shown in Fig. 8.

\subsection{Flavor and charge ratios}

The primary source of electron neutrinos is muon decay, which also gives an equal contribution of muon neutrinos. For $E_{\mu}>2 \mathrm{GeV} / \cos \theta$ muons on average reach the ground before they decay. For $\theta>70^{\circ}$ the curvature of the Earth limits the pathlength in the atmosphere to a few hundred $\mathrm{km}$ and muons with $E_{\mu}>30 \mathrm{GeV}$ typically reach the ground before decaying. For $E_{\nu}$ more than $\sim 100 \mathrm{GeV} / \cos \theta$, the contribution from muon decay practically vanishes and the ratio of $\nu_{e} / \nu_{\mu}$ approaches a small value $\lesssim 5 \%$, with most of the $\nu_{e}$ coming from $K_{e 3}$ decays. Somewhere above a $\mathrm{TeV}$, charm decay dominates $\nu_{e}$.

Atmospheric neutrinos constitute the primary background, as well as a useful calibration source, for neutrino telescopes. The characteristic angular and energy dependences of the fluxes of $\nu_{e}$ and $\nu_{\mu}$ described here should be useful for calibration. As an example, Fig. 9 shows the ratio of horizontal to vertical neutrinos for two flavors from Ref. 21] The angular asymmetry of muon neutrinos increases steadily with energy to a ratio $\sim 2$ in the $\mathrm{TeV}$ range. The large horizontal to verti- 
cal ratio for $\nu_{e}$ and its energy-dependence above $100 \mathrm{GeV}$ should be a distinctive calibration signature in detectors large enough to measure the low rate of $\nu_{e}$-induced cascades.

The ratio $\nu_{\mu} / \bar{\nu}_{\mu}$ is also of interest. As magnetized detectors such as MINOS 40 begin to operate, it will be possible to measure the charge ratio of atmospheric neutrino-induced muons. At the high energies the kaon contribution will be of great importance for evaluating the $\nu / \bar{\nu}$ ratio, which, together with the cross sections $\sigma_{\nu}>$ $\sigma_{\bar{\nu}}$, determines the muon charge ratio. Because the contribution of $\nu_{\mu}$ is larger than that of $\bar{\nu}_{\mu}$, $\mu^{+} / \mu^{-}<1$.

\subsection{Prompt neutrinos from charm}

Leptonic decays of short-lived charmed hadrons gives rise to a third term (not shown) on the right side of Eq. 2, of the same form as the term for the kaon contribution. The spectrumweighted moment $Z_{N \rightarrow \text { charm }}$ is much smaller, but $\epsilon_{\text {charm }} \gtrsim 10^{7} \mathrm{GeV}$, so this component dominates the neutrino spectrum at sufficiently high energy. There are significant uncertainties in inclusive cross sections for hadronic production of charm and hence for the prompt neutrino flux. 411 Because of the steep cosmic-ray energy spectrum, charm production in the forward fragmentation region is likely to make an important contribution to the flux of prompt leptons, in addition to production via central QCD processes. Fig. 10 shows a global view of the spectrum with an estimate of charm production from Ref. 42]. In this quarkparton recombination model, charm becomes the dominant source of $\nu_{e}$ for $E_{\nu} \gtrsim 2 \mathrm{TeV}$ and of $\nu_{\mu}$ for $E_{\nu} \gtrsim 100 \mathrm{TeV}$. Prompt neutrinos have an isotropic angular distribution. The increasing importance of prompt $\nu_{e}$ contributes to the rapid decrease of the ratio of horizontal to vertical electron neutrinos above $100 \mathrm{GeV}$ in Fig. 9.

Acknowledgments I thank M. Honda for collaboration on this work, Todor Stanev for reading the manuscript and Doug Michael and Peter Litchfield for helpful discussions.

\section{REFERENCES}

[1] Y. Fukuda et al., (Super-Kamiokande Collaboration) Phys. Rev. Letters 81 (1998) 1562. See also M. Shiozawa, these Proceedings and C. Mauger, Proc. 31st Int. Conf. on High Energy Physics (Amsterdam, July, 2002) for the complete atmospheric neutrino sample of Super-Kamiokande.

[2] W.W.M. Allison et al. (Soudan Collaboration), Phys. Lett. B391 (1997) 491. and B449 (1999) 137. See also M. Goodman, these Proceedings.

[3] M. Ambrosio et al., Phys. Lett. B 517 (2001) 59.

[4] M. Ambrosio et al., Phys. Lett. B 434 (1998) 451.

[5] Y. Fukuda et al., Phys. Rev. Letters 82 (1999) 2644.

[6] M. Ambrosio et al., Phys. Lett. B 478 (2000) 5.

[7] Y. Fukuda et al., Phys. Lett. B 467 (1999) 185.

[8] S. Fukuda et al., Phys. Rev. Letters 85 (2000) 3999.

[9] T.K. Gaisser \& M. Honda, Ann. Revs. Nucl. Part. Sci. 52 (2002) 153.

[10] Ralph Engel, T.K. Gaisser \& Todor Stanev, Physics Letters B 472 (2000) 113-118.

[11] J.M. Clem \& L.I. Dorman, Space Science Reviews 93 (2000) 335.

[12] Thomas H. Johnson, Phys. Rev. 43 (1933) 834.

[13] L.W. Alvarez \& A.H. Compton, Phys. Rev. 43 (1933) 835.

[14] T. Futagami et al., Phys. Rev. Letters 82 (1999) 5194.

[15] Paolo Lipari, Todor Stanev \& T.K. Gaisser, Phys. Rev. D58 (1998) 073003.

[16] M. Honda, T. Kajita, K. Kasahara \& S. Midorikawa, Phys. Rev. D52 (1995) 4985.

[17]T.K. Gaisser, M. Honda, P. Lipari \& T. Stanev, Proc. 27th ICRC (2001) 1643.

[18] T. Sanuki et al. Ap.J. 545 (2000) 1135.

[19] J. Alcarez et al., Phys. Lett. B490 (2000) 27.

[20] W.R. Webber, R.L. Golden \& S.A. Stephens, Proc 20th Int. Cosmic Ray Conf. 1 (1987) 325.

[21] Vivek Agrawal, T.K. Gaisser, Paolo Lipari \& Todor Stanev, Phys. Rev. D53 (1996) 1314. 
[22] M. Boezio et al. Astrophys. J. 518 (1999) 457. [23] W. Menn et al., Astrophys. J. 533 (2000) 281. [24]E.-S. Seo et al., Astrophys. J. 378 (1991) 763. [25]Ralph Engel, T.K. Gaisser, Paolo Lipari \& Todor Stanev, Proc. 27th ICRC (2001) 1381.

[26] M. Honda, T. Kajita, K. Kasahara \& S. Midorikawa, Proc. 27th Int. Cosmic Ray Conf. 3 (2001) 1162.

[27] S. Roesler, R. Engel \& J. Ranft, Proc. 27th Int. Cosmic Ray Conf. 1 (2001) 439; Phys. Rev. D57 (1998) 2889; Phys. Rev. D55 (1997) 6957.

[28] G. Battistoni, A. Ferrari, T. Montaruli \& P.R. Sala, astro-ph/0207035 and G. Battistoni et al. Astropart. Phys. 12 (2000) 315.
[29] G. Barr for the HARP Collaboration Proc. 27th Int. Cosmic Ray Conf. (2001) 1585.

[30] P. Lipari, Astropart. Phys. 14 (2000) 153.

[31] M. Honda, T. Kajita, K. Kasahara \& S. Midorikawa, Phys.Rev. D64 (2001) 053011.

[32] Y. Tserkovnyak, R.J. Komar, C.W. Nally \& C.E. Waltham, Proc. 27th ICRC (2001) 1196.

[33] J. Wentz, et al., Proc. 27th ICRC (2001) 1167 and private communication.

[34] V. Plyaskin, Phys.Lett. B516 (2001) 213. Revised: hep-ph/0103286, v3 (May 18, 2002).

[35] J. Alcarez et al., Phys. Lett. B 472 (2000) 215.

[36] S. Treiman, Phys. Rev. 91 (1953) 957.

[37] P. Zuccon, et al., astro-ph/0111111.

[38] Paolo Lipari, Astropart. Phys. 14 (2000) 171.

[39]T.K. Gaisser, Cosmic Rays and Particle Physics (Cambridge University Press, 1990).

[40] D. Michael, these Proceedings.

[41] C.G.S. Costa, Astropart. Phys. 16 (2001) 193.

[42] E.V. Bugaev et al., Phys. Rev. D58 (1998) 054001 . 


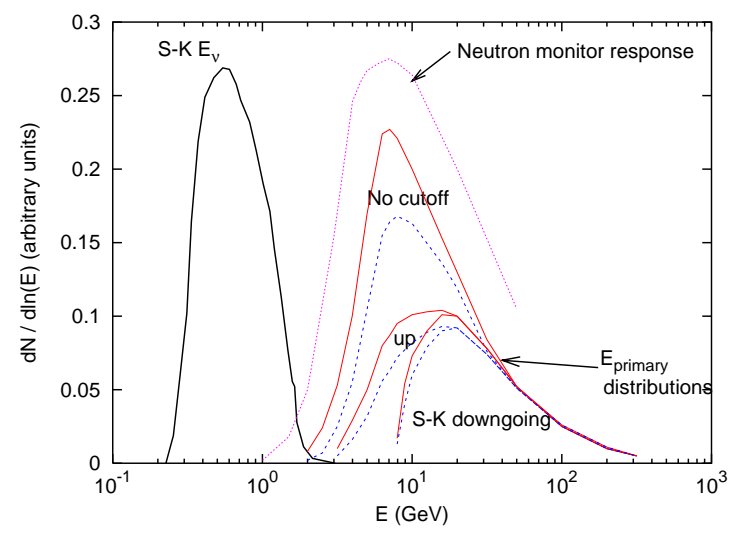

Figure 2. Response functions for sub-GeV neutrinos under several conditions (see text). The three pairs of curves show response functions for the distribution of neutrino energies in the solid curve ( $\mathrm{S}-\mathrm{K} E_{\nu}$ ), which is the same as the left-most curve of Fig. 1.

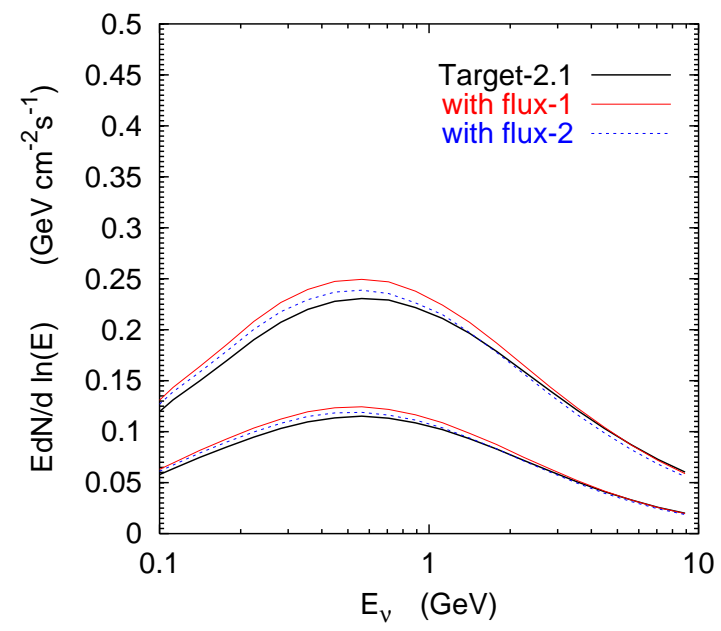

Figure 3. Comparison of neutrino flux for three primary spectra for the location of Kamioka.



Figure 4. Comparison of neutrino flux for three primary spectra for the location of Soudan.

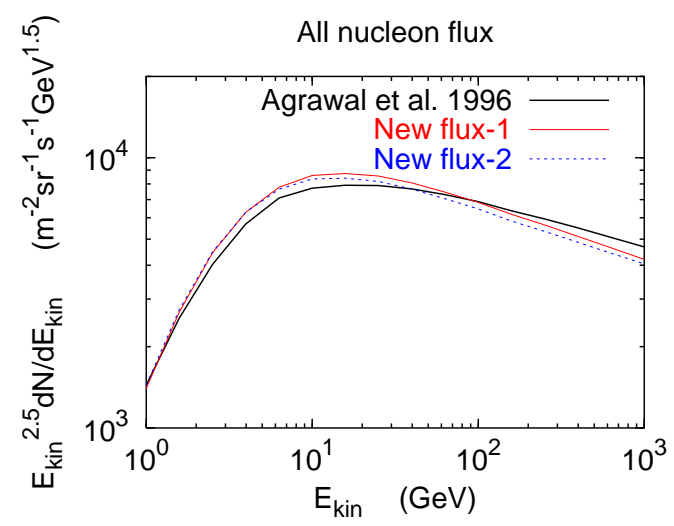

Figure 5. Three fits to the spectrum of cosmic-ray nucleons. 




Figure 6. Comparison of atmospheric neutrino flux calculations for the location of Kamioka.

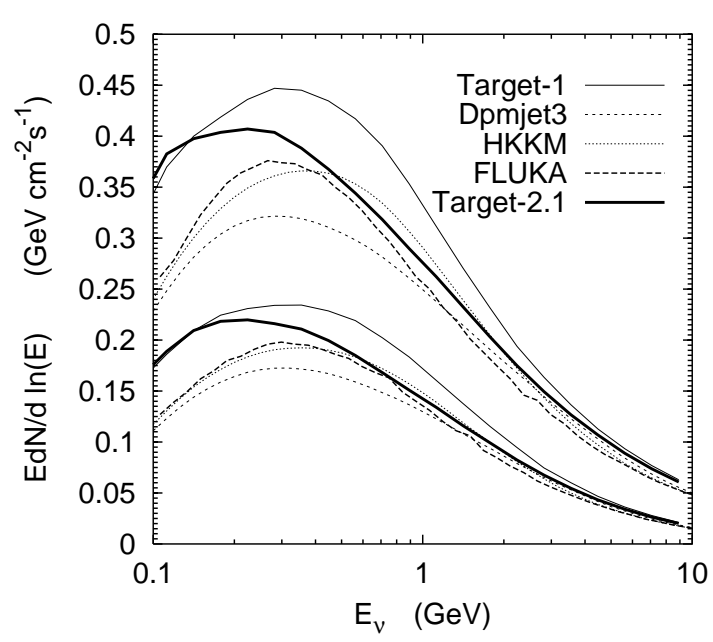

Figure 7. Comparison of atmospheric neutrino flux calculations for the location of Soudan.

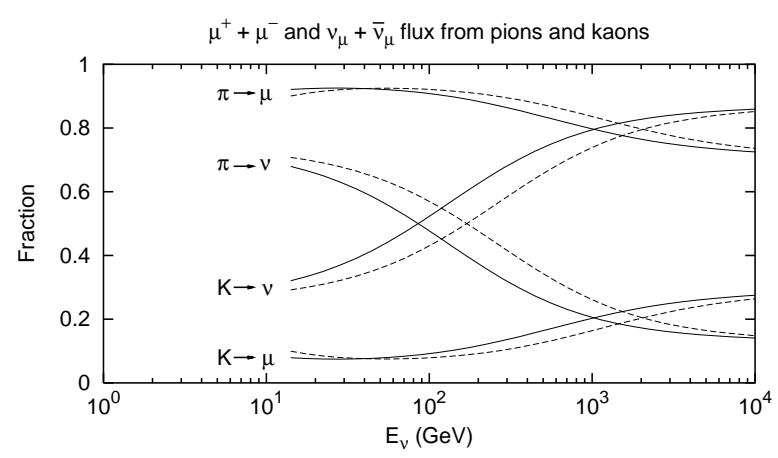

Figure 8. Fraction of atmospheric muons and neutrinos from pions and kaons. Solid: vertical; dashed: $60^{\circ}$.

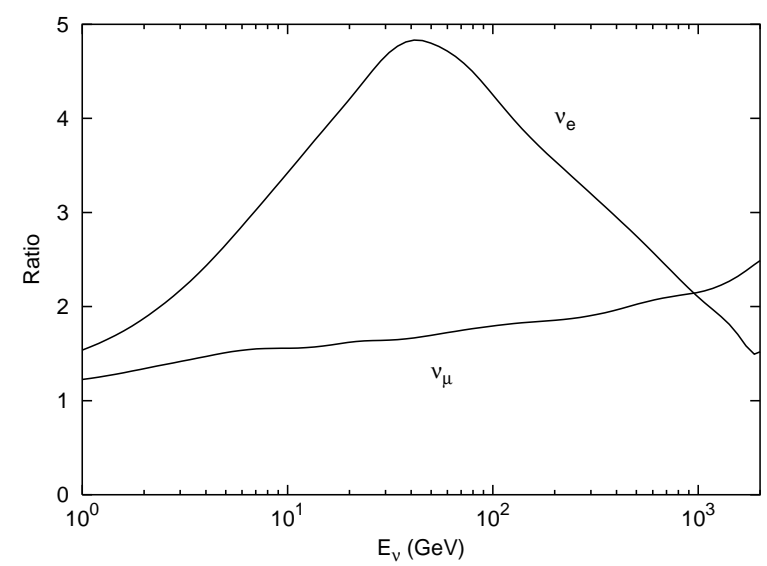

Figure 9. Ratio of horizontal $(0 .<|\cos \theta|<$ $0.375)$ to vertical $(0.675<|\cos \theta|<1$.) atmospheric neutrinos. 


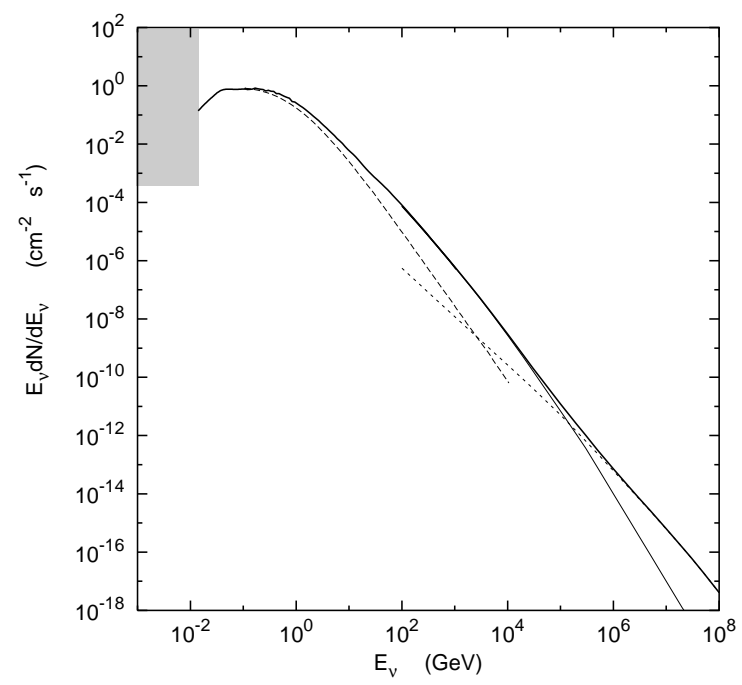

Figure 10. Global view of the atmospheric neutrino spectrum integrated over all directions. The upper solid line shows the total flux of $\nu_{\mu}+\bar{\nu}_{\mu}$ including the effects of oscillations. Above $100 \mathrm{TeV}$ this flux is dominated by prompt neutrinos. Broken lines are $\nu_{e}+\bar{\nu}_{e}$, with the crossover to prompt around $1 \mathrm{TeV}$. Shaded area indicates solar neutrinos. 